\title{
A Methodology for Calculating the Contribution of Exogenous Variables to ARIMAX Predictions
}

\author{
Hao Wang ${ }^{\dagger}{ }^{*}$, Raymond $\mathrm{Yao}^{\dagger}, \mathrm{Likun}_{\mathrm{Hou}}^{\dagger}$, Jie Zhao ${ }^{\dagger}$, Xing Zhao ${ }^{\dagger}{ }^{*}$ \\ $\dagger$ SAP HANA Core, Shanghai
}

\begin{abstract}
Autoregressive integrated moving average with exogenous variables (ARIMAX) is a prevailing model in time series forecasting, yet little attention has been paid to explain the predictions of ARIMAX, which is essential for understanding business behavior and making decisions. Here we argue that the regression coefficients of exogenous variables are not sufficient to measure their contribution to the predictions due to the dynamic nature of the stochastic process in ARIMAX models. In this work, we propose an approach that explains the predictions of ARIMAX in a comprehensive way, by evaluating the effect of the changes in all past values of exogenous variables on the current level of observations, and taking a linear transformation to eliminate the contribution dependence. Through numerical experiments on several datasets, we demonstrate its excellent permformance, good consistency with intuition and much better identification of influential exogenous variables compared with the direct use of regression coefficients.
\end{abstract}

Keywords: Data mining, Time series forecasting, ARIMAX, Exogenous variables, Prediction interpretations

\section{Introduction}

Time series forecasting is at the core of many business processes to enable data-driven decision-making $[1,2]$. Among the existing time series forecasting methods, autoregressive integrated moving average with exogenous variables (ARIMAX) has become of the most popular ones in use [3]. People have combined ARIMAX with many other algorithms to obtain good performance in various needs [4-6]. In practice, ARIMAX uses the past observations and random errors to model the underlying linear dynamics of the focused stochastic process. The incorporation of exogenous variables extended its modeling capability and therefore more satisfactory predictions could be made when involving multiple variables $[7,8]$. The prediction performance is not the only indicator of the goodness of a model, understanding the reason for making predictions is equally important for man to trust the predictions and make decisions. Manufacturers will greatly benefit from knowing which kind of products are most needed and what is the best product mix for maximizing profit. In retail, when determining the location of a new store, one may need to consider the competition from dozens of nearby stores with different styles, distances and main products. In all these scenarios, the importance of exogenous variables (referring to variables which are not affected by others) can be quantized as the contributions they make. For a set of inputs, apart from evaluating the overall contributions from those variables (global understanding), there is also a need to inspect single predictions and their explanations (local interpretations), so that one can make long-term decisions according to global insights and make dynamic adjustments based on real-time changes according to local interpretation. Compared with some complex time-series forecasting methods [9, 10], ARIMAX uses fewer parameters to model the behavior of a stochastic process while maintaining good performance. The relatively simple model structure means that its predictions are easier to

${ }^{*}\{$ hao.wang08,brook.zhao\}@sap.com

This article is () 2021 by author(s) as listed above. The article is licensed under a Creative Commons Attribution (CC BY 4.0) International license (https://creativecommons.org/licenses/by/4.0/legalcode), except where otherwise indicated with respect to particular material included in the article. The article should be attributed to the author(s) identified above. 
explain [11], some previous studies have considered the coefficients as an overall measure of variable importance to the predictions $[12,13]$.

Despite the linear form of exogenous variables in ARIMAX models, the regression coefficients of these variables cannot be viewed as the weights of contribution as the usual way in linear regression. This is because the change in past values of exogenous variables in ARIMAX would also influence the current level of observations. In addition, the effect of variation of one exogenous variable on the current level may also depend on another exogenous variable, due to the collinearity between them. Therefore, the direct use of popular feature attribution methods in interpreting ARIMAX models, such as LIME [14] and SHAP [15] would be problematic.

To address these issues, we propose a method to efficiently explain the predictions of ARIMAX in a faithful way in this paper. The proposed approach establishes a dynamic relationship between the current observation and all the past values of exogenous variables by reformulating the finite lag dependence of the dependent variable to the infinite lag dependence of the exogenous variables. The linear response of dependent variables to the unit change in the values of exogeneous variables shows that due to the collinearity between exogenous variables, the contribution of one exogenous variable may also depend on another exogenous variable. Therefore a linear transformation is performed to make their contributions mutually exclusive. For the numerical analysis, we use the dataset involving electricity consumption of the people of Electrovania. We consider several factors as exogenous variables to train a seasonal ARIMAX model and use the proposed method to decompose the level of observations to the contribution of each exogenous variable. Subsequently, we get the ranking of importance of all variables, as well as a summary of the contribution range corresponding to their specific values. Experimental results indicate that the proposed method effectively identifies the influential exogenous variables and conforms to human intuition.

In short, the main contributions of this paper are summarized as follows:

1) We propose ways to significantly enhance the interpretability of ARIMAX models. The regression coefficients of exogenous variables are proved to be insufficient to measure the contributions in ARIMAX models.

2) The dynamic nature of ARIMAX model is considered when computing the individual contribution of exogenous variables.

3) We extend the method to adapt to multicollinearity between exogenous variables.

4) We offer visualization methods for local interpretations and provide a way of global understanding of an ARIMAX model's behavior.

\section{Preliminaries}

ARIMA model is widely used for the analysis of a class of stochastic processes $[16$, 17]. The Box-Jenkins procedures composed of model identification, model estimation and diagnosis check are proposed for the application of ARIMA in the study of time series [18]. A time series $y_{t}$ with a seasonal $\operatorname{ARIMA}(p, d, q)(P, D, Q)_{s}$ process is generally expressed as:

$$
\phi(B) \Phi\left(B^{s}\right)(1-B)^{d}\left(1-B^{s}\right)^{D} y_{t}=\theta(B) \Theta\left(B^{s}\right) \varepsilon_{t},
$$

where $\varepsilon_{t} \sim W N\left(0, \sigma^{2}\right)$ stands for a white noise with zero mean and constant variance $\sigma^{2}, s$ denotes the length of the seasonal cycle, the backshift operator $B$ is defined by $B y_{t}=y_{t-1}$, $d(D)$ is the order of differencing(seasonal differencing), $\phi(B)$ is the autoregressive operator written as $\phi(B)=1-\phi_{1} B-\phi_{2} B^{2}--\phi_{p} B^{p}$, the moving average operator $\theta(B)$ is defined as $\theta(B)=1-\theta_{1} B-\theta_{2} B^{2}--\theta_{q} B^{q}, \Phi\left(B^{s}\right)$ and $\Theta\left(B^{s}\right)$ are just seasonal autoregressive operator and seasonal moving average operator respectively, their expressions can be obtained by replacing $B$ with $B^{s}, p$ with $P$ and $q$ with $Q$ in $\phi(B)$ and $\theta(B)$. For the sake of simplicity, 
all seasonal parts in Eq. (2.1) will be ignored below, which has no effect on the results of our discussion and can be easily added back at any time.

ARIMA model will be extended into ARIMAX model if one includes $m$ exogenous variables $\mathbf{x}_{t}=\left[x_{t}^{1}, x_{t}^{2},, x_{t}^{m}\right]^{T}$ into the model [19],

$$
\phi(B) \tilde{y}_{t}=\sum_{i=1}^{m} \beta_{i} \tilde{x}_{t}^{i}+\theta(B) \varepsilon_{t},
$$

where $\tilde{y}_{t}$ and $\tilde{x}_{t}^{i}$ for $i=1,2, m$ are stationary series after differencing $\left(\tilde{y}_{t}=(1-B)^{d} y_{t}, \tilde{x}_{t}^{i}=\right.$ $\left.(1-B)^{d} x_{t}^{i}\right), \beta_{i}$ for $i=1,2,, m$ is the regression coefficient of $\tilde{x}_{t}^{i}$.

Yet another form called regression with ARMA error is also proposed to address ARIMA with exogenous variables [20], which can be briefly written as,

$$
\tilde{y}_{t}=\sum_{i=1}^{m} \beta_{i} \tilde{x}_{t}^{i}+\eta_{t}, \phi(B) \eta_{t}=\theta(B) \varepsilon_{t}
$$

where the regression error $\eta_{t}$ follows an ARMA process.

\section{Proposed Method}

The regression coefficients $\beta$ s in Eq. (2.2) and Eq. (2.3) have totally different meanings. In Eq. (2.3), exogenous variables $\tilde{\mathbf{x}}_{t}=\left[\tilde{x}_{t}^{1}, \tilde{x}_{t}^{2}, \tilde{x}_{t}^{m}\right]^{T}$ only contributes to $y_{t}$ at the given time $t$, while in Eq. (2.2), the changes occurred in historical periods of $\tilde{\mathbf{x}}_{t}$ will be propagated to current time and also have contributions to $y_{t}$, due to the autoregression on the left side of Eq. (2.2). That is to say, $\beta \mathrm{s}$ in Eq. (2.2) actually are the coefficients of regression of $\tilde{y}_{t}$ conditionally on $\tilde{\mathbf{x}}_{t}$ and the previous $\tilde{y}_{t-1}, \tilde{y}_{t-2},, \tilde{y}_{t-p}$, which makes it difficult for them to have an intuitive interpretation like those in Eq. (2.3).

However, in many cases, one would like to deal with exogenous variables in the way of Eq. (2.2) instead of Eq. (2.3), since from Eq. (2.2) one can know exactly in which way $\tilde{y}_{t}$ is related to the previous values of $\tilde{\mathbf{x}}_{t}$. Taking advertising as an example, a company uses some form of advertising to draw new customers over a period. It is important to know what role the ads placed in the past few days play in daily sales, which day will contribute the most, and how slowly the sales will drop to the average when ads are stopped in the future. More requirements could be found frequently in business promotions, intervention analysis, capacity plainning, etc.. A full treatment of the lagged exogenous variables in time series will lead to dynamic regression models [21], but the solving is quite cumbersome and the discussion is beyond the scope of this paper.

\subsection{Dynamic Nature of ARIMAX Models}

In the following, we will decompose the contribution to $\tilde{y}_{t}$ from each exogenous variable in Eq. (2.2), without relying on the previous values of $\tilde{y}_{t}$. We first reformulate Eq. (2.2) by dividing by $\phi(B)$ on both sides such that no lagged $\tilde{y}_{t}$ exist on the left-hand side,

$$
\tilde{y}_{t}=\phi^{-1}(B) \sum_{i=1}^{m} \beta_{i} \tilde{x}_{t}^{i}+\phi^{-1}(B) \theta(B) \varepsilon_{t} .
$$

We are only interested in the first term on the right-hand side of Eq. (3.1). By using Wold representation of a stationary process [22], it could be repressed as,

$$
\Delta_{x} \tilde{y}_{t}=\phi^{-1}(B) \sum_{i=1}^{m} \beta_{i} \tilde{x}_{t}^{i}=\sum_{i=1}^{m} \beta_{i} \sum_{j=0}^{\infty} \nu_{j} \tilde{x}_{t-j}^{i} .
$$

where $\nu_{j}$ for $j=0,1, t, t$ is now the coefficient measuring how $\tilde{y}_{t}$ responds to a unit change in $\tilde{x}_{t-j}$. As an example, we show below the behavior of model with two autoregressive lags 
$(\operatorname{AR}(2)), \phi(B)=1-\phi_{1} B-\phi_{2} B^{2}$. The transfer function of $\phi^{-1}(B)$ is given by replacing $B$ with $z^{-1}$ and taking the inverse of $\phi(B)$,

$$
h(z)=\frac{1}{1-\phi_{1} z^{-1}-\phi_{2} z^{-2}},
$$

where $z$ is a complex variable. Assuming $r_{1}$ and $r_{2}$ are the two roots of equation $z^{2}-\phi_{1} z-$ $\phi_{2}=0$. Then the amplitude-frequency response is

$$
\begin{array}{r}
H\left(e^{j \omega}\right)=\mid \frac{1}{r_{1}-r_{2}}\left(\frac{r_{1}}{1-r_{1} \cdot e^{j \omega}-r_{1}^{*} \cdot e^{-j \omega}+r_{1} \cdot r_{1}^{*}}\right. \\
\left.-\frac{r_{2}}{1-r_{2} \cdot e^{j \omega}-r_{2}^{*} \cdot e^{-j \omega}+r_{2} \cdot r_{2}^{*}}\right) \mid,
\end{array}
$$

where $*$ represents the conjugate of a complex number, $j$ is the imginary unit and $\omega$ the frequency in unit of rad.

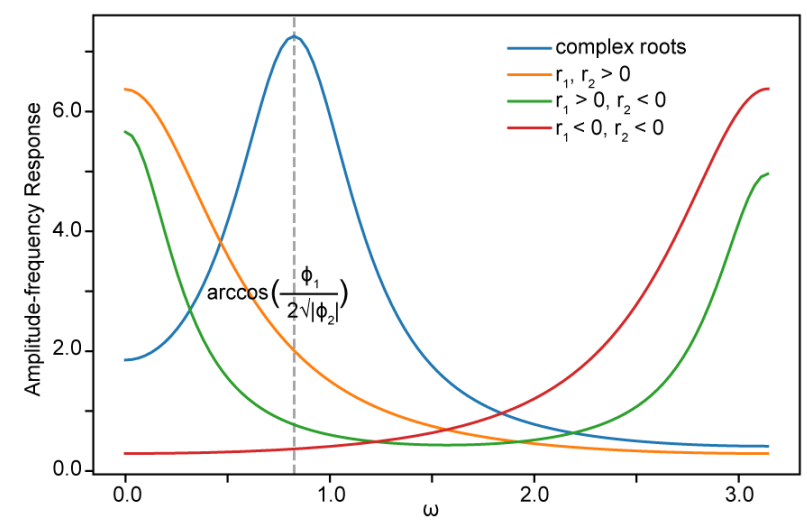

Figure 1. Amplitude-frequency response of $\left(1-\phi_{1} B-\phi_{2} B^{2}\right)^{-1}$. The blue line shows the response when $r_{1}$ and $r_{2}$ are complex conjugate roots, and the vertical dashed line indicates the peak at $\omega_{0}=\arccos \left(\phi_{1} / 2 \sqrt{\left|\phi_{2}\right|}\right)$. The orange, green and red lines represent the response when $r_{1}$ and $r_{2}$ are real roots. They display peaks at either $\omega=0, \omega=\pi$ or both.

Fig. 1 depicts the graph of $\left|H\left(e^{j \omega}\right)\right|$. When $r_{1}$ and $r_{2}$ are real numbers $\left(\phi_{1}^{2}+4 \phi_{2} \leq 0\right)$, its amplitude-frequency response behaves like just the $\mathrm{AR}(1)$ process, only displaying peaks at $\omega=0$ and/or $\omega=\pi$, as shown by the orange, green and red lines. The blue line shows peak at $\omega_{0}=\arccos \left(\phi_{1} / 2 \sqrt{\left|\phi_{2}\right|}\right)$ when the two roots are complex numbers. This means that the variation of exogenous variables at time $t$ will propagate over time, its response will reach extreme values every $\Delta_{t}=\pi / \omega_{0}$ and drop off gradually. After some time, the response will become so small that it cannot be distinguished from the random error.

\subsection{Collinearity Between Exogenous Variables}

In addition, because there may be collinearity between exogenous variables, attention needs to be paid in attributing the individual contributions to the coefficients $\nu_{j}$ in Eq. (3.2). In this case, variation in one exogenous variable will simultaneously affect other exogenous variables. Denoting the covariance matrix of $\tilde{\mathbf{x}}_{t}$ as $\boldsymbol{\Sigma}$, the effect of variation in $\tilde{x}_{t}^{i}$ for $i=1,2,, m$ on the future series $\tilde{y}_{t+k}(k \geq 0)$ can be quantified as,

$$
\frac{\partial \tilde{y}_{t+k}}{\partial\left[\tilde{x}_{t}^{1}, \tilde{x}_{t}^{2}, \ldots, \tilde{x}_{t}^{m}\right]^{T}}=\nu_{k} \tilde{\boldsymbol{\Sigma}} \cdot \boldsymbol{\beta}
$$


where $\tilde{\boldsymbol{\Sigma}}$ denotes the result of dividing each row of $\boldsymbol{\Sigma}$ by the variance of the corresponding exogenous variable in the denominator, and $\beta=\left[\beta_{1}, \beta_{2}, \cdots, \beta_{m}\right]^{T}$ is the vector of regression coefficients. Details of the derivation of the partial differencing in Eq. (3.3) are given in Appendix A.

To eliminate the correlations between exogenous variables, we can take a linear transformation of $\tilde{\mathbf{x}}_{t}$. The Cholesky decomposition of $\boldsymbol{\Sigma}$ gives $\boldsymbol{\Sigma}=\mathbf{L L}^{T}$, where $\mathbf{L}$ is a lower triangular matrix. If we let

$$
\tilde{\boldsymbol{\delta}}_{t}=\mathbf{L}^{-1} \tilde{\boldsymbol{x}}_{t}
$$

then the identity covariance matrix of $\tilde{\boldsymbol{\delta}}_{t}$ can be obtained.

Now, the contributions to $\tilde{y}_{t}$ in Eq. (3.2) can be readily expressed as,

$$
\Delta_{x} \tilde{y}_{t}=\nu(B) \boldsymbol{\beta}^{T} \cdot \mathbf{L} \mathbf{L}^{-1} \tilde{\boldsymbol{x}}_{t}=\overline{\boldsymbol{\nu}}(B) \tilde{\boldsymbol{\delta}}_{t},
$$

where $\overline{\boldsymbol{\nu}}(B)=\overline{\boldsymbol{\nu}}_{0}+\overline{\boldsymbol{\nu}}_{1} B+\overline{\boldsymbol{\nu}}_{2} B^{2}+, \cdots$, and $\overline{\boldsymbol{\nu}}_{i}=\nu_{i} \boldsymbol{\beta}^{T} \cdot \mathbf{L}$ for $i=0,1,, \infty, \nu_{i}$ has been defined in Eq. (3.2). Theoretically the total number of terms in $\overline{\boldsymbol{\nu}}(B)$ can be infinite, but the coefficients would always approximately decay to zero with finite order in a stationary process. Without loss of generality, lets set the truncated order of $\overline{\boldsymbol{\nu}}(B)$ as $l$, and view the coefficients of all terms with orders higher than $l$ to be 0 . Then the expansion of $\overline{\boldsymbol{\nu}}(B)$ will result in a transformation matrix $\mathbf{V}$ of size $l \times m$, so the total contirbution

$$
\Delta \mathbf{Y}=\mathbf{V} \otimes \mathbf{D}
$$

can be obtained, where $\mathbf{D}$ is the matrix for all exogenous variables, which can possibly contain values in training phase and prediction phase. $\otimes$ denotes a kind of convolution operator, meaning for $j=1,2, m$, taking the $j$-th column of $\mathbf{V}$ and the $j$-th row of $\mathbf{D}$ for convolution, and performing a vector summation on the $m$ results to get the total contributions.

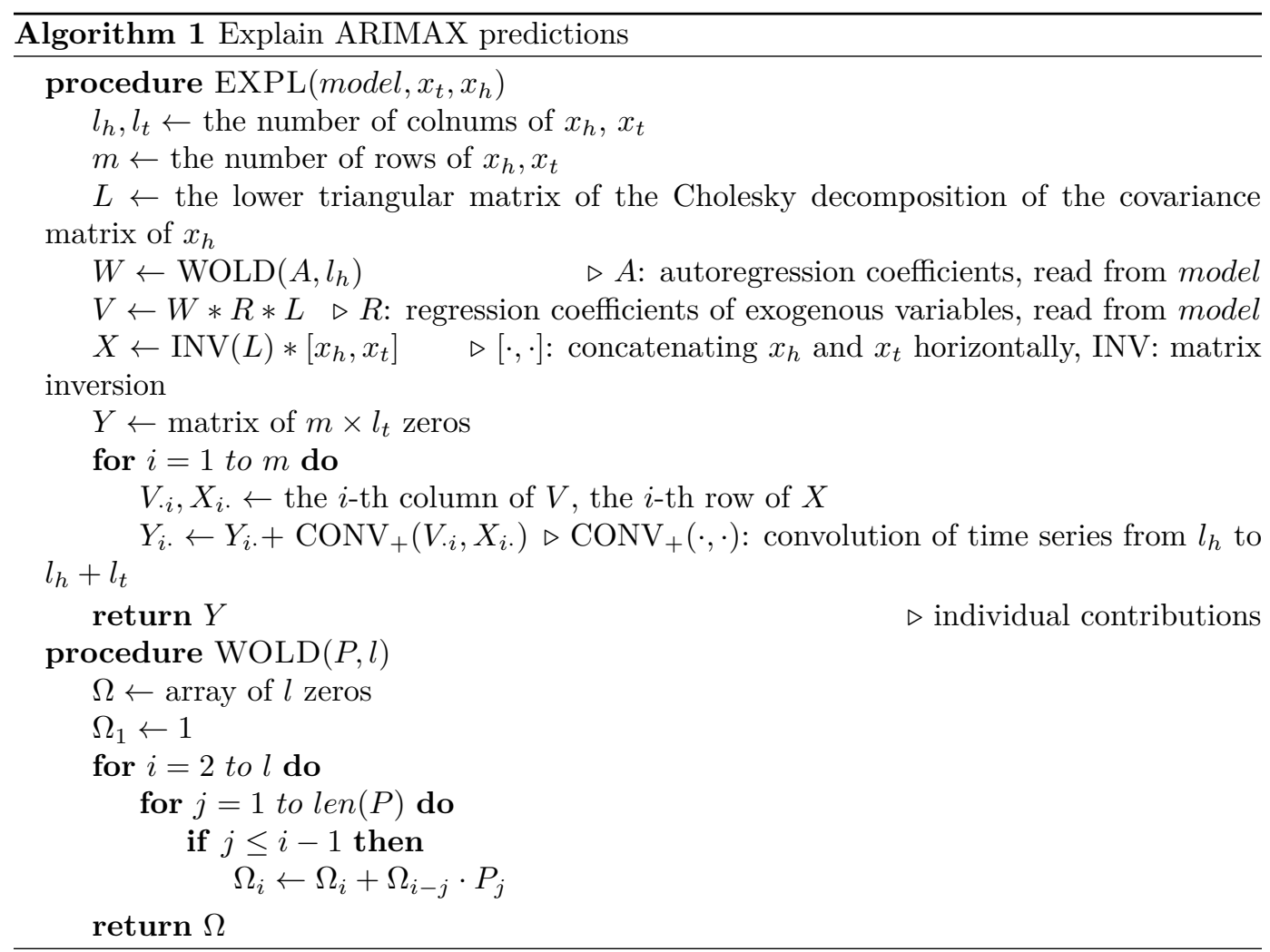


Algorithm 1 summarizes the pseudo-code used to calculate the individual contribution of exogenous variables in ARIMAX predictions. Its inputs consist of an ARIMAX model, an instance expressed as a vector of values of exogenous variables at time $t$ and all its previous values within the length of $l$. The procedure WOLD in the algorithm presents the calculation of $\nu_{i}$ for $i=1,2, \infty$ in Eq. (3.2), while the procedure EXPL recaps the calculation of Eq. (3.4) and Eq. (3.5). It returns the final individual contributions. In EXPL, the maximal value of time lags $l_{h}$ cannot be larger than the length of time series $T$, and a fast Fourier transform algorithm can be used to calculate the convolution, so our algorithm runs in $O(m T \log T)$ time and $O(T)$ memory.

\section{Experimental Results And Discussion}

In this section, numerical experiments are carried out to examine the effectiveness and interpretability of the proposed method. We first present and discuss the results of the contribution of exogenous variables for the electricity consumption ${ }^{1}$ dataset. Then we include more datasets and empirically compare the results of the proposed method and the most widely used feature attribution method on them.

\subsection{Model Training And Local Interpretations for Electricity Consumption Dataset}

The ARIMAX model Eq. (2.2) has been applied in the forecast of electricity consumption. For the electricity consumption of the people of Electrovania, the hourly time series of the electricity consumption in the first 23 days of each month from July 2013 to June 2017 was given. Temperature, anonymized feature var1, pressure, wind speed and anonymized feature var2, which were considered to possibly affect the consumption, were regarded as exogeneous variables. We have encoded the categorical variable var2 with conjugated Bayesian encoding, instead of dummy encoding in the modeling. To validate the accuracy of the predictions, we trained with data between 0:00 on October 1, 2016 and 23:00 on October 21, 2016, and the data used to forecast was from 0:00 on October 22, 2016 to 23:00 on October 23, 2016.

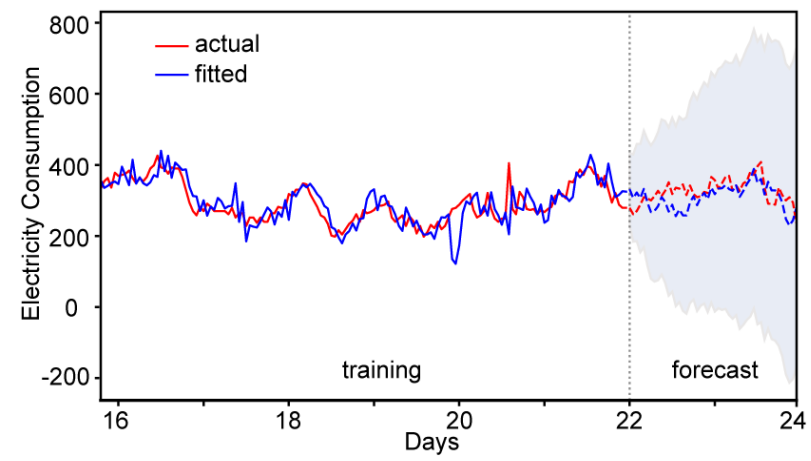

Figure 2. Time series of electricity consumption. The vertical dashed line separates the training period from the forecast period. The red line represents the actual training data (solid line) and test data (dashed line), while the blue line shows the fitted values (solid line) and the predictions (dashed line). A 95\% confidence interval is drawn shaded in gray within the prediction range. The unit of electricity consumption is MWh.

Predictive Analysis Library (PAL) in SAP HANA was used to establish the ARIMAX model. $\mathrm{PAL}^{2}$ includes a wealth of machine learning algorithms for predictive analysis and

\footnotetext{
${ }^{1}$ Data: https://www.kaggle.com/utathya/electricity-consumption

${ }^{2}$ Help document: https://help.sap.com/viewer/319d36de4fd64ac3afbf91b1fb3ce8de/2020_03_QRC/en-US
} 
data mining, which can be called from SAP HANAs SQLScript or python API (hanaml) ${ }^{3}$. To begin with, the autocorrelation functions showed a seasonal cycle with length of $168(=24 \times 7)$, that is, a weekly cycle, In this case, a seasonal component would be included in the model. To make the time series stationary, a first-order differencing $(1-B)$ and a first-order seasonal differencing $\left(1-B^{168}\right)$ were performed to both the dependent variable (electricity consumption) and exogenous variables. Heuristic search was performed to select out the best order of parameters. During each step, an interdependent least squares estimation and exact maximum likelihood estimation were done with the Kalman filter. Then based on the minimal values of the goodness of fit values AICc (corrected Akaike Information Criterion), a seasonal $\operatorname{ARIMAX}(1,1,2)(0,1,0) 168$ model was chosen from all the candidates. The diagnosis check validated that the error term is a white noise process. Finally, we predicted based on the trained model, Fig. 2 shows the fitted result (blue solid line), predictions (blue dashed line) and also a 95\% confidence interval (in gray shade). Compared with actual electricity consumption (red lines), the performance of the selected ARIMAX model is satisfactory.

(a)

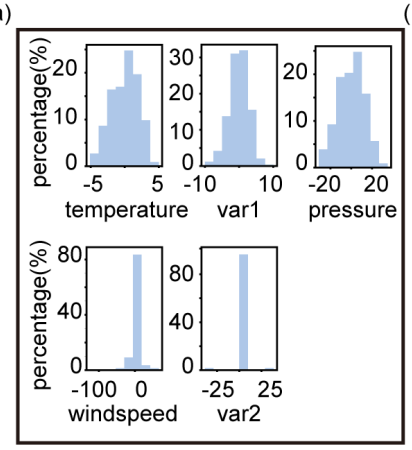

(b)

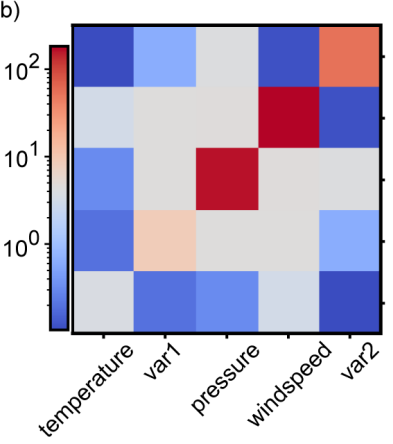

(c)

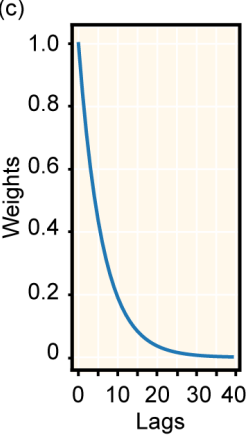

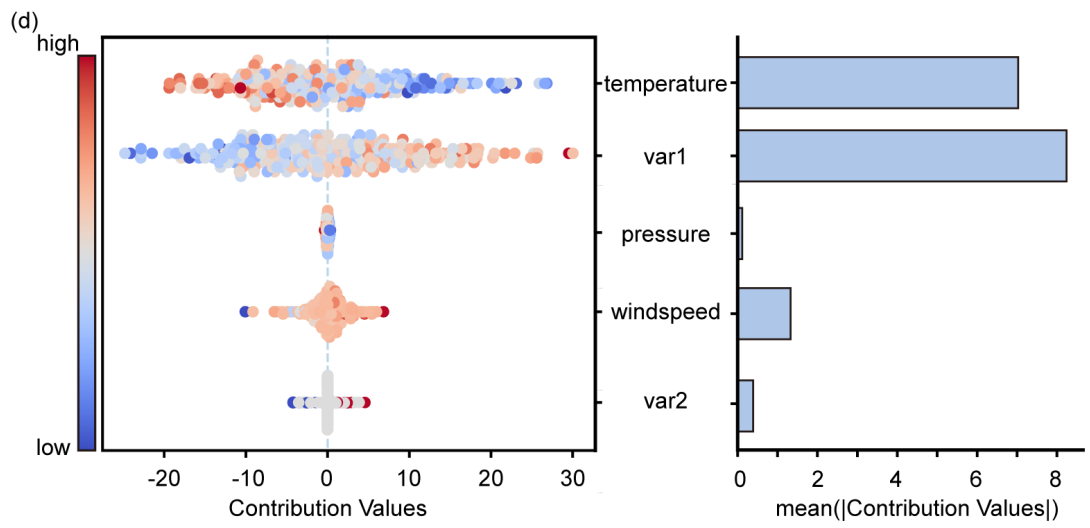

Figure 3. (a) Combining histograms displaying the distributions of exogenous variables after differencing. (b) Heatmap of covariance matrix of exogeneous variables. Annotations on the bottom show the name of the variables. The anti-diagonal elements are their variance. (c) Plot of transfer function versus time lag. (d) Left panel: summary plot of the contributions of the five exogenous variables. The x-axis is the contribution values. Each dot is a time point, which is colored by the values of variables. The redder the color, the larger the value, the bluer the smaller, and the medium value will be gray. Right panel: bar chart of the average contribution value magnitude.

ARIMAX models allow the impact of all the past and current values of the exogenous variables on the dependent variable, so their contributions should be added up to obtain

\footnotetext{
${ }^{3}$ Help document: https://pypi.org/project/hana-ml
} 
the total contribution for current time. In the seasonal ARIMAX $(1,1,2)(0,1,0)_{168}$ model, the transfer function of $\left(1-\phi_{1} B\right)^{-1}$ helps to assign contribution weights to all time lags. As shown in Fig. 3(c), as the lag increases, the weights decay with power law and become so small that can be ignored when the lag is greater than 35 . More precisely in this AR(1) process, the weights would be less than $\phi_{1}^{34} \approx 0.0034$ when the lags are greater than 35 .

If the correlation between exogenous variables is strong, we need to be careful about computing the contribution weights. In Fig. 3(a), var1 and temperature have nearly symmetric distributions centered at zero, the change of their value is much smaller than that of pressure, wind speed and var2. Fig. 3(b) shows the heatmap of the covariance matrix of exogenous variables. the anti-diagonal elements are the variance of each variables. Obviously, pressure, wind speed and var2 have larger variances. Compared with other variables, wind speed has stronger collinearity with others, so its contribution is underestimated if the regression coefficients is regarded as the contribution weight. All the collinearity can be eliminated using the linear transformation Eq. (3.4) so the true contributions from individual variables could be obtained. Eq. (3.5) was used to evaluate the total contribution at each timestamp. The summary plot of contributions under all training time and the bar chart of the average magnitude were given by Fig. 3(d). The top two contributions to the regression were attributed to var1 and temperature. They dominate the total contribution (the right panel of Fig. 3(d)). The dot diagram (the left panel of Fig. 3(d)) shows that for temperature, the more negative the value, the greater the positive contribution, which can be understood as in the coming winter, the electricity consumption will rise if temperature drops (blue dots) and the consumption will drop if temperature rises (red dots). However, var1 just has the opposite trend of the contribution, negative values (blue dots) and values near the mean (gray dots) tend to have a negative impact on consumption, while positive values contribute positively to that. The total contribution of wind speed reduced to about one quarter of var1 due to the elimination of collinearity, and its local contribution is almost zero most of the time. Fig. 3(d) is a good example that local interpretations are used as building blocks to get a global understanding of the behavior of an ARIMAX model.

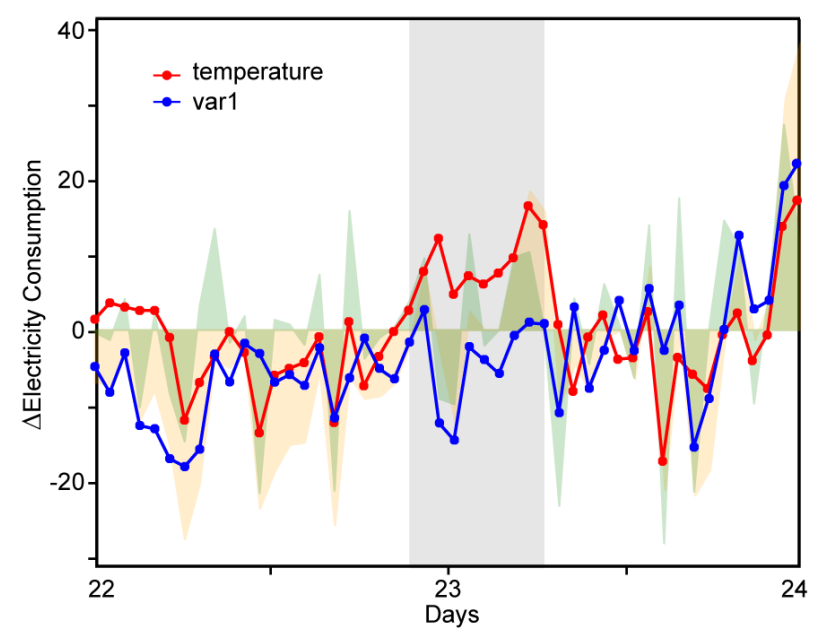

Figure 4. Individual contribution of temperature (red line) and var1 (blue line) to ARIMAX predictions. Within this range, we plot the total contribution of all external variables including all times in the past (in orange shade) and the total contribution only at current time (in green shade).

Local interpretation can provide accurate, real-time explanations that are critical for decision-making. The greater a variable contributes to the prediction, the more important 
it is for decision-making. In addition, the variables that play a leading role at different timestamps may be different and may not always contribute the most during the entire time period. As shown in Fig. 4, from 0:00 to 20:00 on the 22nd day, the negative contribution of var1 is greater than that of temperature, but from 21:00 on the 22nd to 6:00 on the 23rd, the temperature dropped rapidly and causes an increase in electricity consumption, so the total change of consumption during this period was positive. Then after the time until 23:00 on the $23 \mathrm{rd}$, the two variables together determined the change in electricity consumption level. There is a clear discrepancy between the contribution of exogenous variables of current day (green shade in Fig. 4) and the cumulative contribution of all the past days based on their weights (orange shade in Fig. 4), which indicates that the past changes of exogenous variables can propagate over time and influence the value of current dependent variable. Therefore, to interpret the prediction accurately, it is necessary to take the contribution of all the past time into account.

\subsection{Examining Performance Compared with Linear SHAP}

To further validate the proposed algorithm, we select another two datasets walmart sales ${ }^{4}$ and workplace mobility ${ }^{5}$, besides the forementioned electricity consumption dataset. In the experiments, we compare the proposed method with linear SHAP, one of the most widely used feature attribution methods which only relies on the regression coefficients and more details about linear SHAP can be found in [15]. We calculate the individual contribution of each feature for every timestamp and sum them up to get the total contribution of exogenous variables.

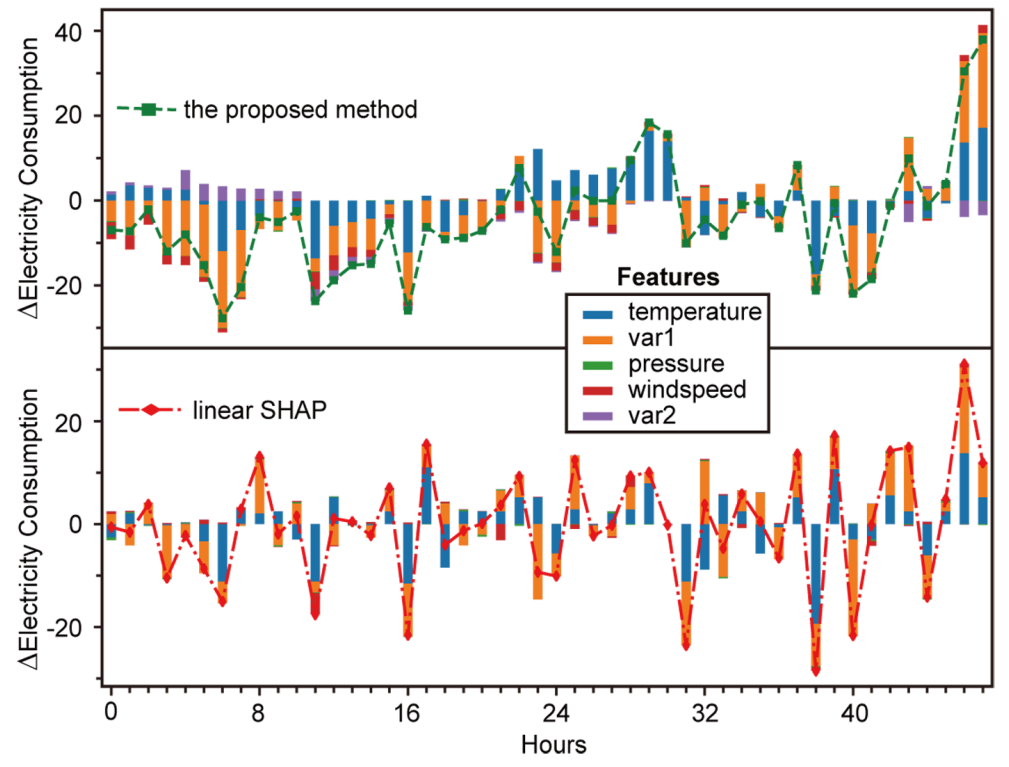

Figure 5. Stacked bar chart for individual contribution of features in electricity consumption dataset using the proposed method (above) and linear SHAP (below). The dashed and dotted lines respectively represent the total contribution with these two methods.

\section{- Electricity Consumption Dataset}

In Fig. 5, the proposed method and linear SHAP show a similar pattern, that is, in general the contributions of temperature and var1 are dominant, although the relative

\footnotetext{
${ }^{4}$ Data: https://www.kaggle.com/c/walmart-recruiting-store-sales-forecasting

${ }^{5}$ Data: https://www.kaggle.com/datatattle/predictworkplacemobility
} 
proportions of the two are not the same. The contribution of wind speed in the proposed method is more significant than that in linear SHAP, which is reasonable if we observe that wind speed has a strong correlation with temperature and var1 but the correlation is ignored in linear SHAP.
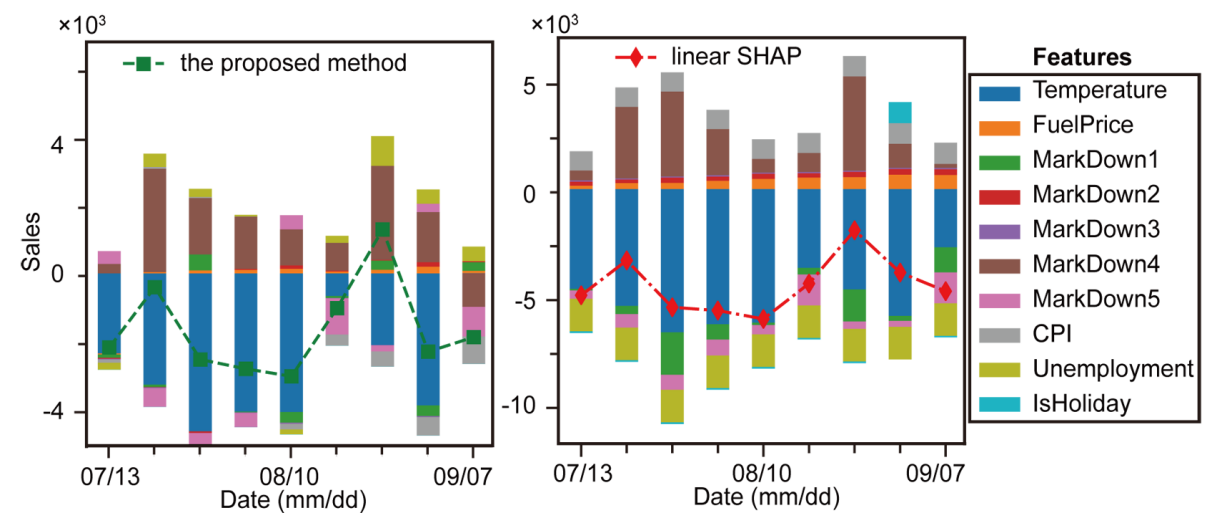

Figure 6. Stacked bar chart for walmart sales dataset.

\section{- Walmart Sales Dataset}

In the experiment, the weekly sales data of department 1 in store 1 are selected spanning from Feburary 5, 2012 to September 7, 2012. We train with data between Feburary 5th and July 6th and use the data from July 13th to September 7th to compare the two methods. As shown in Fig. 6, 10 features are considered in the model. temperature and MarkDown4 contribute the most in the two methods. However, linear SHAP is not sensitive to the changes in feature values and it tends to give relatively similar individual contributions at different timestamp. This is because linear SHAP doesn't consider the dynamic nature of ARIMAX model. There is also a violation of common sense that CPI (Consumer Price Index) has a relatively large impact on sales in linear SHAP. CPI is a long-term economic indicator which cannot have sustained influence on short-term sales. The proposed method takes into account all past contributions for current time and therefore does not have the above problems.

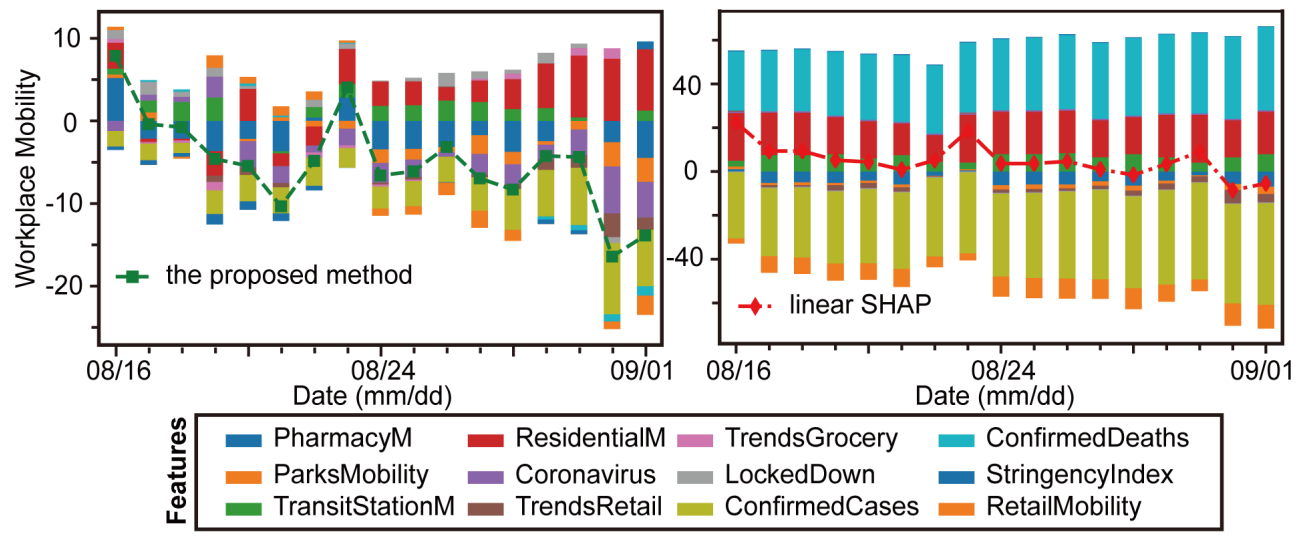

Figure 7. Stacked bar chart for workplace mobility dataset.

\section{- Workplace Mobility Dataset}


For workplace mobility dataset, we use the first 183 days as traning data and decompose contributions into individual features on the remaining 17 days. Fig. 7 shows 12 features that might be influential to workplace mobility. In this experiment, linear SHAP gives even more strange interpretations. In addition to the inability to accurately capture the change in the contributions of features over time, linear SHAP regards confirmed cases and confirmed deaths as two featuers that have completely opposite effects on workplace mobility. This is inconsistent with common sense, an increased number of confirmed deaths can only lead to a decrease in workplace mobility, not the other way around. In the proposed method, residential mobility, Corona virus and confirmed cases are considered to have the greatest impact on residential mobility most of the time, in line with our intuitions.

\section{Conclusion}

Explaining predictions using the contribution of individual exogenous variables is particularly important in ARIMAX. They measure the influence of the change of exogenous variables on the predicted values and uncover an intuitive interpretation of why the model makes such predictions, which can be even more important than the performance of models predictions to understand business behaviors and make decisions. Using regression coefficients as feature importance in ARIMAX is incomplete, since they may either overestimate or underestimate the true impact of variables. In contrast, we proposed the approach that accumulates the contributions from past values with calculated weights and eliminates collinearity between exogenous variables, which makes it efficient and feasible for local interpretation, and further provides global understanding of the behavior of an ARIMAX model. Experiments show that it can well identify influential exogenous variables and deeply reveal how exogenous variables affect the predictons. It is particularly valuable and interesting to apply this method to explain predictions and help decision-making in a wide range of fields such as finance, supply chain management, advertising, marketing and other areas that make predictions based on ARIMAX models. This is the topic of our further work.

\section{Acknowledgements}

We would like to acknowledge the support of SAP devX and SAP HANA.

\section{Appendix A. Derivation of partial difference $\frac{\partial \tilde{y}_{t+k}}{\partial\left[\tilde{x}_{t}^{1}, \tilde{x}_{t}^{2}, \ldots, \tilde{x}_{t}^{m}\right]^{T}}$}

Proof. Lets assume that any pair $\left(\tilde{x}_{t}^{i}, \tilde{x}_{t}^{j}\right)$ drawn from $\left[\tilde{x}_{t}^{1}, \tilde{x}_{t}^{2},, \tilde{x}_{t}^{m}\right]$ would be distributed as a bivariate Gaussian. Then the expectation of $\tilde{x}_{t}^{i}$ condition on $\tilde{x}_{t}^{j}, E\left(\tilde{x}_{t}^{i} \mid \tilde{x}_{t}^{j}\right)$ can be written as,

$$
E\left(\tilde{x}_{t}^{i} \mid \tilde{x}_{t}^{j}\right)=\tilde{\mu}_{i}+\frac{\operatorname{Cov}\left(\tilde{x}_{t}^{i}, \tilde{x}_{t}^{j}\right)}{\sigma_{j}^{2}}\left(\tilde{x}_{t}^{j}-\tilde{\mu}_{j}\right),
$$

where $\tilde{\mu}_{i}$ and $\tilde{\mu}_{j}$ are the mean values of $\tilde{x}_{t}^{i}$ and $\tilde{x}_{t}^{j}$ over the whole time range, $\operatorname{Cov}(\cdot, \cdot)$ is the covariance between $\tilde{x}_{t}^{i}$ and $\tilde{x}_{t}^{j}$, and $\sigma_{j}^{2}$ is the variance of $\tilde{x}_{t}^{j}$. Base on the assumption, we obtain the result of the partial difference,

$$
\begin{aligned}
\frac{\partial \tilde{y}_{t+k}}{\partial\left[\tilde{x}_{t}^{1}, \tilde{x}_{t}^{2}, \ldots, \tilde{x}_{t}^{m}\right]^{T}} & =\nu_{k}\left[\frac{\partial \tilde{x}_{t}^{i}}{\partial \tilde{x}_{t}^{j}}\right]_{i=1, \cdots, m ; j=1, \cdots, m} \cdot\left[\beta_{1}, \beta_{2}, \cdots, \beta_{m}\right]^{T} \\
& =\nu_{k} \tilde{\boldsymbol{\Sigma}} \cdot \boldsymbol{\beta}
\end{aligned}
$$




\section{References}

[1] O. B. Sezer, M. U. Gudelek, and A. M. Ozbayoglu. "Financial time series forecasting with deep learning: A systematic literature review: 2005-2019". In: Applied Soft Computing 90 (2020), p. 106181.

[2] C. Faloutsos, J. Gasthaus, T. Januschowski, and Y. Wang. "Forecasting big time series: old and new". In: Proceedings of the VLDB Endowment 11.12 (2018), pp. 2102-2105.

[3] J. D. Hamilton. Time series analysis. Princeton university press, 2020.

[4] G. P. Zhang. "Time series forecasting using a hybrid ARIMA and neural network model". In: Neurocomputing 50 (2003), pp. 159-175.

[5] N. I. Sapankevych and R. Sankar. "Time series prediction using support vector machines: a survey". In: IEEE Computational Intelligence Magazine 4.2 (2009), pp. 24-38.

[6] C. S. Bojer and J. P. Meldgaard. "Kaggle forecasting competitions: An overlooked learning opportunity". In: International Journal of Forecasting (2020).

[7] M. Brabec, O. Konár, E. Pelikán, and M. Malỳ. "A nonlinear mixed effects model for the prediction of natural gas consumption by individual customers". In: International Journal of Forecasting 24.4 (2008), pp. 659-678.

[8] W. Anggraeni, R. A. Vinarti, and Y. D. Kurniawati. "Performance comparisons between arima and arimax method in moslem kids clothes demand forecasting: Case study". In: Procedia Computer Science 72 (2015), pp. 630-637.

[9] S. Hochreiter and J. Schmidhuber. "Long short-term memory". In: Neural computation 9.8 (1997), pp. 1735-1780.

[10] D. Salinas, V. Flunkert, J. Gasthaus, and T. Januschowski. "DeepAR: Probabilistic forecasting with autoregressive recurrent networks". In: International Journal of Forecasting 36.3 (2020), pp. 1181-1191.

[11] K. Aas, M. Jullum, and A. Løland. "Explaining individual predictions when features are dependent: More accurate approximations to Shapley values". In: Artificial Intelligence (2021), p. 103502.

[12] S. Tao, J. Corcoran, F. Rowe, and M. Hickman. "To travel or not to travel:Weatheris the question. Modelling the effect of local weather conditions on bus ridership". In: Transportation research part C: emerging technologies 86 (2018), pp. 147-167.

[13] S. Chadsuthi, C. Modchang, Y. Lenbury, S. Iamsirithaworn, and W. Triampo. "Modeling seasonal leptospirosis transmission and its association with rainfall and temperature in Thailand using time-series and ARIMAX analyses". In: Asian Pacific journal of tropical medicine 5.7 (2012), pp. 539-546.

[14] M. T. Ribeiro, S. Singh, and C. Guestrin. "" Why should i trust you?" Explaining the predictions of any classifier". In: Proceedings of the 22nd ACM SIGKDD international conference on knowledge discovery and data mining. 2016, pp. 1135-1144.

[15] S. Lundberg and S.-I. Lee. "A unified approach to interpreting model predictions". In: Proceedings of the 31st International Conference on Neural Information Processing Systems. 2017, pp. 4768-4777.

[16] P. J. Brockwell and R. A. Davis. "Time Series: Theory and Methods, (Springer Series in Statistics)". In: (2009).

[17] C. W. J. Granger and P. Newbold. Forecasting economic time series. Academic Press, 2014.

[18] G Box and G. M. Jenkins. "Time series analysis: forecasting and control. Holden dsy". In: Inc. San Francisco (1970).

[19] G. E. Box, G. M. Jenkins, G. C. Reinsel, and G. M. Ljung. Time series analysis: forecasting and control. John Wiley \& Sons, 2015.

[20] R. J. Hyndman and G. Athanasopoulos. Forecasting: principles and practice. OTexts, 2018.

[21] A Pankratz. "Forecasting with dynamic regression models. New York: John Wiley". In: (1991).

[22] H. Wold. "Causality and econometrics". In: Econometrica: Journal of the Econometric Society (1954), pp. 162-177. 